\title{
CARTA SINE LITTERIS. ENEA SILVIO PICCOLOMINI UND DIE URKUNDENPRAXIS IM FRÜHMITTELALTER
}

\section{Tamás Nótári*}

Enea Silvio Piccolomini ${ }^{1}$ erzählt in seinem Werk De Europa, das er noch als Kardinal im Jahre 1458 beendet hat, im 65 Kapitel, wo er sich mit Kärnten auseinandersetzt, eine interessante Geschichte - ihrer Literaturgattung nach eine Legende - über einen Herzog namens Ingo, der während der Regierungszeit Karl des Großen gelebt haben sollte. Der Legende nach gab der Herzog im Jahre 790 seinen Untertanen ein Festmahl, bei dem die anwesenden Bauern aus silbernem und goldenen Gefäßen speisten, während die Vornehmen ihr Mahl nur aus Tonschüsseln zu sich nehmen konnten. Sein Verhalten erklärte der Herzog damit, dass die Seelen der in Hütten

1 Zum Leben und Wirken des Enea Silvio Piccolomini siehe G Voigt Enea Silvio de1 Piccolomini als Papst Pius der Zweite und sein Zeitalter (Berlin, 1856-1863); W Boulting Aeneas Sylvius (Enea Silvio de' Piccolomini - Pius II): Orator, Man of Letters, Statesman and Pope (London, 1908); CM Ady Pius II, The Humanist Pope (London, 1913); E Hocks Pius II. und der Halbmond (Freiburg im Breisgau, 1941); G Bürck Selbstdarstellung und Personenbildnis bei Enea Silvio Piccolomini (Pius II) (Basel-Stuttgart, 1956); RJ Mitchell The Laurels and the Tiara: Pope Pius II, 1458-1464 (London, 1962); PJ Weinig Aeneam suscipite, Pium recipite: Aeneas Silvius Piccolomini: Die Rezeption eines humanistischen Schriftstellers im Deutschland des 15. und 16. Jahrhunderts (Wiesbaden, 1998).

* Wissenschaftlicher Hauptmitarbeiter, Rechtswissenschaftliches Institut des Gesellschaftswissenschaftlichen Zentrums der Ungarischen Akademie der Wissenschaften; Universitätsdozent, Károli Gáspár Universität Budapest.

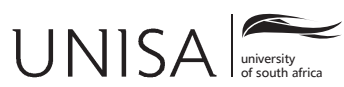


wohnenden, aber getauften Bauern weiß und rein, die der in Palästen lebenden, und Götzenbilder anbetenden Adeligen aber schwarz und unrein seien. Der Herzog habe das Gastmahl nach diesem Prinzip veranstaltet. Beschämt waren die Adeligen unter der Leitung von Arn und Virgil, den Bischöfen von Salzburg scharenweise zum Taufbecken geströmt:

Fama est anno septimgentesimo nonagesimo post Christi Salvatoris ortum imperante Carolo Magno ducem gentis, Ingonem nomine, ingens convivium provincialibus praeparasse et agrestibus quidem, ad conspectum suum intromissis, in vasis aureis atque argenteis, nobilibus vero ac magnatibus, procul ab oculis collocatis, fictilibus ministrare iussisse. Interrogatum, cur ita faceret, respondisse non tam mundos esse, qui urbes et alta palatia quam qui agros et humiles casas colerent. Rusticis, qui Christi evangelium accepissent, baptismatis unda purificatis candidas et nitidas esse animas; nobiles ac potentes, qui spurcitias idolorum sequerentur, sordidas ac nigerrimas. Se vero pro animarum qualitatibus instruxisse convivium. Castigatos ea re nobiles catervatim sacri baptismatis undam quaerentes brevi tempore sub Vergilio et Arnone iuvavensibus episcopis universos Christi fidem accepisse. ${ }^{2}$

Es stellt sich mit Recht die Frage, ob Enea Silvio diese biblische, bzw parabolische Geschichte von irgendeinem anderen Autor übernommen hat; und wenn ja, von wem. Die Frage scheint prima facie einfach beantwortbar zu sein: Die Erzählung stammt aus der Conversio Bagoariorum et Carantanorum, die anlässlich des Prozesses des päpstlichen Legaten und Erzbischofs von Sirmium, Methodius verfasst wurde. Der Slawenapostel Methodius wurde 870 auf der in Anwesenheit Ludwigs des Deutschen abgehaltenen Regensburger Synode unter Mitwirkung des Salzburger Erzbischofs Adalwin und seinen Bischöfen angeklagt und verurteilt. Ob die Conversio als Anklage- oder als eine Legitimationsschrift des Prozesses im Nachhinein verfasst wurde, ist nicht zu ermitteln. Dieses Werk beinhaltet ebenso eine Erzählung, deren Hauptfigur Ingo ist:

Simili modo etiam Arn episcopus successor sedis Invavensis deinceps curam gessit pastoralem, undique ordinans presbyteros et mittens in Sclavinam, in partes videlicet Quarantanas atque inferioris Pannoniae, illis ducibus atque comitibus, sicut pridem Virgilius fecit. Quorum unus Ingo vocabatur, multum carus populis et amabilis propter suam prudentiam. Cui tam oboediens fuit omnis populus, ut, si cuique vel carta sine litteris ab illo directa fuit, nullus ausus est suum neglegere praeceptum. Qui etiam mirabiliter fecit: Vere servos credentes secum vocavit ad mensam, et qui eorum dominabantur infideles, foris quasi canes sedere fecit ponendo ante illos panem et carnem et fusca vasa cum vino, ut sic sumerent victus. Servis autem staupis deauratis propinare iussit. Tunc interrogantes primi deforis dixerunt: 'Cur facis nobis sic?'At ille: 'Non estis digni non ablutis corporibus cum sacro fonte renatis communicare, sed foris domum ut canes sumere victus.' Hoc facto fide sancta instructi certatim cucurrerunt baptizari. Et sic deinceps religio christiana succrescit. ${ }^{3}$

2 Aeneas Sylvius De Europa 65.

3 Conversio Bagoariorum et Carantanorum 7. 
In der vorliegenden Studie, nachdem die Entstehung der Conversio Bagoariorum et Carantanorum skizzenhaft geschildert (1) und die Anfänge der Salzburger Karantanenmission kurz dargestellt worden sind - Piccolominis Erzählung nach sollte Ingo ein christlicher Herzog Karantaniens gewesen sein - (2), wird es versucht auf folgende Fragen eine Antwort zu finden. In wie fern kann der von Piccolomini erwähnte und Jahrhunderte lang in seiner Existenz nicht angezweifelte Herzog Ingo als eine reale historische Person betrachtet werden? (3) Was konnte die in der Conversio erwähnte carta sine litteris bedeuten haben, und warum wurde dieses Motiv nicht von Enea Silvio übernommen, wo es doch zur Bekräftigung der Autorität des Ingos hätte verwendet werden können? (4) Aus welcher Quelle stammte die Erzählung von Ingos Gastmahl, und welche Rolle spielte diese Geschichte in der Conversio? (5) Übernahm Enea Silvio diese Parabel direkt aus der Conversio, oder soll es in diesem Fall mit einer vermittelnden Schrift, bzw. mit einem Mittelsautor gerechnet werden? (6)

1. Die Hauptinteressenten der Slawen- und Awarenmission im 9 Jahrhundert waren vorwiegend Byzanz und das Frankenreich, letzteres konnte sich bei der Ausführung der Missionsarbeit auf das Erzbistum Salzburg und auf das ziemlich unabhängige Politik betreibende Patriarchat von Aquileia stützen. Dieses Gleichgewicht wurde durch die eigenständige Missionspolitik von Rom ins Schwanken gebracht. ${ }^{4}$ Dieser dreiseitige Zusammenstoß von Interessen lag dem Zwist zwischen dem Slawenapostel Methodius und dem Erzbistum Salzburg zugrunde. Der aus Byzanz stammende Missonar, später päpstlicher Legat und Erzbischof von Sirmium, Methodius wurde im Jahre 870 auf der Regensburger Synode in Anwesenheit von Ludwig dem Deutschen von dem Salzburger Erzbischof Adalwin und den bayerischen Bischöfen verurteilt, weil er durch seine Missionstätigkeit in Pannonien, die laut der Überzeugung der bayerischen Kirche seit über siebzig Jahren der Jurisdiktion von Salzburg unterstand, die Interessen des Erzbistums Salzburg gefährdet, und seine Gerichtsbarkeit in Frage gestellt haben sollte. Methodius wurde zwei Jahre lang in Gefangenschaft gehalten, und kam nur dank einer energischen Intervention des Papstes Johannes VIII. frei. ${ }^{5}$ Es ist nicht zu ermitteln, ob die

4 K Reindel „Bayern vom Zeitalter der Karolinger bis zum Ende der Welfenherrschaft (788-1180)“ in Handbuch der bayerischen Geschichte (München, 1981) Bd 1 S 249-349.

5 H Löwe Der Streit um Methodius: Quellen zu den nationalkirchlichen Bestrebungen in Mähren und Pannonien im 9. Jahrhundert (sl, 1948), S 5-62; K Bosl „Probleme der Missionierung des böhmisch-mährischen Herrschaftraumes“ in M Hellmann, R Olesch, B Stasiewski \& F Zagiba (Hrsg) Cyrillo-Methodiana. Zur Frühgeschichte des Christentums bei den Slaven 863-1963 (Graz, 1964) S 1-38; M Schellhorn „Erzbischof Adalwin von Salzburg und die Pannonische Mission" Mitteilungen der Gesellschaft für Salzburger Landeskunde (1964) 104 S 103-121; H Dopsch „Slawenmission und päpstliche Politik: Zu den Hintergründen des Methodios-Konfliktes“ in Th Piffl-Perčević \& A Stirnemann (Hrsg) Der heilige Method, Salzburg und die Slawenmission (Innsbruck-Wien, 1987) S 303-340; M Eggers Das Erzbistum des Method: Lage, Wirkung und Nachleben der kyrillomethodianischen Mission (München, 1996) S 19-44. 
Conversio Bagoariorum et Carantanorum als Anklageschrift oder als Dokument, das den Prozess später zu legitimieren hatte, verfasst wurde. ${ }^{6}$

Das Darstellungsprinzip der Conversio wurde von Hans-Dieter Kahl höchst treffend mit folgenden Worten charakterisiert:

Was da getrieben wird, ist nichts anderes als ein waghalsiges Spiel dicht an der Grenze der Wahrheit, gerade noch unanfechtbar für den, der Bescheid weiß, dem Unkundigen jedoch abweichende Kombinationen offenlassend, ja nahelegend, die den Zwecken der Denkschrift ungleich besser entgegenkamen. Man ahnt einen wohlunterrichteten Gewährsmann, der jedoch sehr wohl weiß, was er will, was nicht, und man bedauert, daß er von seinen Kentnissen keinen besseren Gebrauch gemacht hat. Raffiniertes Verschweigen unerwünschter oder gar „gefährlicher" Zusammenhänge und Fakten, ähnlich raffinierte Zusammenziehung von Ereignissen, die womöglich weit auseinanderlagen - das sind auch sonst die Hauptmittel, die der Verfasser für seinen Zweck einsetzt. ${ }^{7}$

Herwig Wolfram nahm in seiner Ausgabe aus dem Jahre 1979 aufgrund des 14 Kapitels der Conversio 871 als Entstehungsjahr an, aber in seiner Monografie aus 1995 zog die Differenzen der heutigen und der mittelalterlichen Zeitrechnung in Betracht, und sprach sich für das Entstehungsjahr 870 aus. Fritz Lošek schloss sich in seiner Edition aus 1997 Wolframs Datierung aus 1995 an. ${ }^{8}$ Über die Person des Verfassers können aufgrund einiger Passagen des Werkes Vermutungen angestellt werden, die Möglichkeit einer eindeutigen Identifikation muss jedoch ausgeschlossen werden. Herwig Wolfram machte die ziemlich zurückhaltende, mehrfach relativierte Aussage, dass der auctor - in diesem Falle vielleicht nicht der Verfasser, sondern der Auftragsgeber des Werkes - Erzbischof Adalwin gewesen sein könnte. ${ }^{9}$ Es mag von Interesse sein, dass in der Conversio nur zwei Personen das Epitheton piissimus tragen, und zwar König Ludwig der Deutsche (cap 12) und Erzbischof Adalwin von Salzburg (cap 9), dh möglicherweise der Adressat und der Auftraggeber des Werkes. Mit ziemlicher Sicherheit ist aber aus der Formulierung des 5 Kapitels zu ermitteln, dass der Autor ein Bayer oder ein Salzburger sein sollte. ${ }^{10}$

6 Dazu siehe A Lhotsky Quellenkunde zur mittelalterlichen Geschichte Österreichs (Graz, 1963) S 155-157.

7 H-D Kahl „Virgil und die Salzburger Slawenmission“ in H Dopsch \& R Juffinger (Hrsg) Virgil von Salzburg, Missionar und Gelehrter (Salzburg, 1985) S 112-121, 112.

8 H Wolfram Conversio Bagoariorum et Carantanorum: Das Weißbuch der Salzburger Kirche über die erfolgreiche Mission in Karantanien und Pannonien (Wien, 1979) S 15, 141; F Lošek Die Conversio Bagoariorum et Carantanorum und der Brief des Erzbischofs Theotmar von Salzburg (Hannover, 1997) S 6.

9 H Wolfram, Salzburg, Bayern, Österreich: Die Conversio Bagoariorum et Carantanorum und die Quellen ihrer Zeit (Wien-München, 1995) S 197. Vgl F Lošek „Sieben Fragen zu sieben ausgewählten lateinischen Denkmälern des Salzburger Frühmittelalters: Gemeinsamkeiten und Unterschiede" in L Kolmer \& Chr Rohr (Hrsg) Tassilo III. von Bayern: Großmacht und Ohnmacht im 8. Jahrhundert (Regensburg, 2005) S 121-136, $124 \mathrm{ff}$.

10 Conversio Bagoariorum et Carantanorum 5 ... orta seditione, quod carmula dicimus. Vgl Lex Baiuvariorum 2, 3. 
2. Als erstes slawisches Volk kamen die Karantanen mit dem bayerischen Herzogtum und dem Frankenreich in Berührung, da Fürst Boruth um 741/742 die Bayern gegen die Awaren um militärische Hilfe bat. Der Sieg über die Awaren begründete die bayerische Herrschaft über Karantanien. ${ }^{11}$ Um 743 wurde Fürst Boruth gezwungen den Bayern seinen Sohn Cacatius und seinen Neffen Cheitmar als Geisel zu übergeben, die vom Salzburger Priester Lupo eine Unterweisung im christlichen Glauben erhielten. ${ }^{12}$ Laut dem 4. Kapitel der Conversio ließen nach Boruths Tod die Bayern auf den Druck der Franken und die Bitte der Karantanen den bereits zum Christentum bekehrten Cacatius in sein Heimatland zurückkehren, wo er als Herrscher eingesetzt wurde. Cacatius verstarb aber nach drei Jahren und Cheitmar (752-769) konnte mit Pippins Erlaubnis das karantanische Fürstentum übernehmen. Der Priester Lupo gab seinem Patensohn Cheitmar seinen in Salzburg zum Priester geweihten Neffen, Maioranus als Begleiter zur Seite, der seiner Aufgabe eifrig nachkam den jungen Fürsten an seine christlichen Pflichten und den gegenüber dem Salzburger Kloster gebührenden Gehorsam zu erinnern. Dieses Verhältnis versucht die Conversio aus den schon erwähnten politischen Gründen als eine Art Vasallentum darzustellen. ${ }^{13}$ Aufmerksamkeit verdient jedoch, dass in der Entlassung der späteren Fürsten Cacatius und Cheitmar der fränkische Einfluss eine erhebliche Rolle gespielt hat, und dass weder Lupo, noch Maioranus - beide einfache Priester - auf Geheiß des Salzburger Abtbischofs Virgil (749-784) ihre Ratgebertätigkeit an der Seite des Karantanenfürsten ausgeübt haben. Hätte eine engere Verbindung zwischen den Karantanenfürsten, den Priestern Lupo und Maioranus und dem Salzburger Bistum bestanden, hätte der Verfasser der Conversio diese Tatsache mit Sicherheit nicht unerwähnt gelassen. Es ist daher nicht auszuschließen, dass die ersten Bekehrungsversuche der Karantanen nicht von der bayerischen Kirche, sondern von dem Frankenherrscher selbst ausgingen. ${ }^{14}$

Bedeutende Erfolge konnte das Bistum Salzburg in der Karantanenmission erst danach verbuchen, als auf Cheitmars Bitte Bischof Virgil den Chorbischof

11 Wolfram (Fn 8) S 73; S Szádeczky-Kardoss Az avar történelem forrásai 557-töl 806-ig (Die Quellen der Awarengeschichte von 557 bis 806). (Budapest, 1998) S 266f; Schellhorn (Fn 5) S 104; H Dopsch „Rupert, Virgil und die Salzburger Slawenmission“ in 1000 Jahre Ostarrîchi: Seine christliche Vorgeschichte, Mission und Glaube im Austausch zwischen Orient und Okzident (Innsbruck-Wien, 1997) S 88-139, 101ff.; W Pohl „Bayern und seine Nachbarn im 8. Jahrhundert" in L Kolmer \& Chr Rohr (Hrsg) Tassilo III von Bayern: Großmacht und Ohnmacht im 8. Jahrhundert (Regensburg, 2005) S 57-66, 59.

12 Conversio Bagoariorum et Carantanorum 4. Vgl H-D Kahl „Zwischen Aquileia und Salzburg: Beobachtungen und Thesen zur Frage romanischen Restchristentums im nachvölkerwanderungszeitlichen Binnen-Noricum“ in H Wolfram \& F Daim Die Völker an der mittleren und unteren Donau im fünften und sechsten Jahrhundert (Wien, 1980) S 33-81, 44; Kahl (Fn 7) S 116; Dopsch (Fn 11) S 102.

13 Conversio Bagoariorum et Carantanorum 4; Wolfram (Fn 8) S 86; Dopsch (Fn 5) S 307; Dopsch (Fn 11) S 103-107; Schellhorn (Fn 5) S 104.

14 Kahl (Fn 7) S 114; Pohl (Fn 11) S 65. 
Modestus mit der Missionsleitung betraute. Nach dem Tode des Modestus konnte Bischof Virgil die Mission fortsetzen, obwohl die Bekehrungsarbeit von zahlreichen Heidenaufständen (carmula) erschwert und gehindert wurde. Neuen Schwung bekam die Missionstätigkeit, als 772 der Widerstand der Karantanen von Tassilo III mit Waffengewalt endgültig bezwungen wurde. Nachdem Karantanien in das Herzogtum Bayern eingegliedert wurde - obwohl es seine eigenen Fürsten (duces gentis) behalten durfte -, waren alle Hindernisse der von dem Salzburger Kirche geleiteten und unter Mitwirkung des Freisinger Bistums ausgeführten Missionsarbeit aus dem Wege geräumt. ${ }^{15}$ Von einer aktiven Beteiligung des Papsttums an der Bekehrungsarbeit wird zwar expressis verbis nicht berichtet; da aber ein Teil Karantaniens auf dem Gebiet des Patriarchats Aquileia lag, der dort jedoch keine nennenswerte Missionstätigkeit ausübte, kann ein stillschweigendes päpstliches Einverständnis bezüglich der Erweiterung der Herrschaftsgebietes vom Salzburger Bistum angenommen werden. ${ }^{16}$ Als später der Patriarch Ursus Aquileias Rechte bezüglich Karantaniens wieder ins Leben zu rufen versuchte, berief sich der Salzburger Erzbischof Arn (784-821) auf die Privilegien der Päpste Zacharias (741-752), Stefans II (752-757) und Pauls I (757-767), die das Gebiet Karantaniens dem Bistum Salzburg zusprachen. Karl der Große bestimmte die Karantanien durchquerende Drau als Grenzfluss zwischen den beiden Metropolien. ${ }^{17}$ Obwohl die Originalfassungen der besagten päpstlichen Urkunden uns nicht erhalten blieben, kann deren Existenz nicht angezweifelt werden. Einerseits ist die Erlangung dreifacher päpstlicher Bestätigungen der Salzburger Rechtsansprüche auf Karantanien durchaus typisch für die präzise Vorgehensweise Virgils bezüglich seiner Missionspolitik, andererseits hätte es Erzbischof Arn nicht gewagt sich beim Kaiser auf nicht vorhandene Papsturkunden zu berufen, zumal sein Gegner der rechtskundige Patriarch Ursus von Aquileia war. ${ }^{18}$

15 Conversio Bagoariorum et Carantanorum 5; H Wolfram „Das Fürstentum Tassilos III, Herzogs der Bayern“ Mitteilungen der Gesellschaft für Salzburger Landeskunde (1968) 108 S 157-179; Schellhorn (Fn 5) S 105; W Störmer ,Zum Problem der Slawenmission des Bistums Freising im 9. Jahrhundert“ in Th Piffl-Perčević \& A Stirnemann (Hrsg) Der heilige Method, Salzburg und die Slawenmission (Innsbruck-Wien, 1987) S 207-220. Zu Virgils Wirken in Salzburg und seiner Missionstätigkeit siehe H Löwe „Salzburg als Zentrum literarischen Schaffens im 8. Jahrhundert“ Mitteilungen der Gesellschaft für Salzburger Landeskunde (1975) 115 S 99-143; H Wolfram „Virgil als Abt und Bischof von Salzburg“ in H Dopsch \& R Juffinger (Hrsg) Virgil von Salzburg, Missionar und Gelehrter (Salzburg, 1985) S 342-356; J Jahn „Arbeo und Cozroh: Verfassungsgeschichtliche Beobachtungen an bairischen Quellen des 8. und 9. Jahrhunderts“ Mitteilungen der Gesellschaft für Salzburger Landeskunde (1990) 130 S 202-291, 208-226); Wolfram (Fn 9) S 252-275.

16 Dopsch (Fn 5) S 308; zur Salzburger Missionspolitik siehe noch B Wavra Salzburg und Hamburg: Erzbistum und Missionspolitik in karolingischer Zeit (Berlin, 1991) S 157-199.

17 W Hauthaler \& F Martin Salzburger Urkundenbuch (Salzburg, 1916) Bd 2 S 3; Eggers (Fn 5) S 24.

18 Dopsch (Fn 5) S 309. 
3. Ingo trat als Herzog zum ersten Mal im Werk Liber certarum historiarum des Viktringer Abtes Johann (gest um 1345/1347) in der ersten Hälfte des 14 Jahrhunderts in Erscheinung:

\begin{abstract}
Nam anno Domini septingentesimo nonagesimo sub Karolo imperatore et Ingone duce et Vergilio et Arnone episcopis Iuvavensibus Ingo dux nobiles terre et servos eis subiectos ad convivium invitavit et nobiles quidem tamquam canes et immundos deputavit et pane et carnibus foris ab oculis suis pavit et vinum in vasis fuscis propinavit, servos vero vasis splendidis et deauratis in sua presencia collocavit. Et dum quererent nobiles, quid in hoc pretenderet, respondit hos simplices et fideles, mundos et sacro baptismate confirmatos, eos autem immundos atque indignos sine sacri fontis ablucione existere et fedatos. Qui audientes certatim ad baptismum cum fervore fidei cucurrerunt et ergo hoc privilegium honoris et commercium rusticale cum principe non ad nobiles, sed ad simplices usque huc creditur propagatum. ${ }^{19}$
\end{abstract}

Johann von Viktring nahm nebst der Ingo-Sage auch die Geschichte über den Missionsauftrag von Karl dem Großen an Arn, und das Auftreten des Methodius aus dem 12 Kapitel der Conversio über. Im Gebrauch der Conversio als Quelle beschränkte sich jedoch der Autor hauptsächlich auf das Inhaltliche, stilistisch weist der Liber certarum historiarum eine größere Unabhängigkeit zur Conversio auf. ${ }^{20}$ Marcus Hansitz identifizierte Ingo im 18 Jahrhundert mit dem legendären Herzog Domitian. Der Sage nach war der heidnische Herzog Domitian ein vehementer Christenverfolger, es wurde ihm durch Gottes Gnade die Bekehrung zu teil, daraufhin stürzte er die Götzenstatuen selber in den See, und wurde zum heiligen Herzog Karantaniens. ${ }^{21}$ Die Existenz des oft als legendäre Figur angesehenen Domitian könnte durch eine Inschrift aus dem Millstätter Kloster aus dem 9. Jahrhundert belegt werden, wegen den Datierungsproblemen der Inschrift und den inkongruenten Fakten ermahnt Herwig Wolfram mit Recht zur Vorsicht. ${ }^{22}$ Es ist zu erwähnen, dass der für die Annerkennung der historischen Realität und die Kanonisation Domitians eintretende Robert Eisler, hinter dem Namen Domitianus die Form Domislaus, einen Namen slawischen Ursprungs vermutete, und die Aufmerksamkeit auf die mögliche Verbindung zwischen der Legende des Statuen stürzenden Herzogs und der gelehrten Etymologie des Namens von Millstatt (mille statuae) lenkte. ${ }^{23}$

19 Iohannes abbas Victoriensis, Liber certarum historiarum 2, 13.

20 Zu Johann von Viktring siehe Lhotsky (Fn 6) S 293; Lošek (Fn 8) S 52; H Fichtenau „Herkunft und Sprache Johanns von Viktring“ Carinthia (1975) 165 S 25-39; A Lhotsky „Johann von Viktring“" in H Wagner \& H Koller (Hrsg) Aufsätze und Vorträge (Wien, 1970) Bd 1 S 131-148.

21 M Hansiz Germania Sacra (Augsburg, 1729) Bd 2 S 104; R Eisler „Die Legende vom heiligen Karantanenherzog Domitian“ Mitteilungen des Instituts für österreichische Geschichtsforschung (1907) 28 S 52-116, 91.

22 F Nikolasch „Domitian von Millstatt - eine Erfindung des 12. Jahrhunderts?“ Carinthia (1990) 180 S 235-253; F Glaser „Eine Marmorinschrift aus der Zeit Karls des Großen“ Carinthia (1993) 183 S 303-318; Wolfram (Fn 9) S 289. 
Der Großteil der modernen Geschichtsforschung sah in dem Herzog Ingo eine historische Person, und versuchte ihn als Träger eines germanischen aber nichtbayerischen Namens mit jemandem aus den Paralellquellen der Conversio zu identifizieren. ${ }^{24}$ Michael Mitterauer zB identifizierte Ingo mit Etgar aus dem 10 Kapitel der Conversio, einem Karantanenfürst bewiesener Existenz. ${ }^{25}$ Als weiterer Identifikationsversuch ist seine Gleichsetzung mit dem Slawen Woinimir (Wonomyrus Sclavus) zu erwähnen, der auf den Befehl des Fürsten Erich von Friaul im Jahre 795 den awarischen Ring angegriffen hat. Über den Rang und die Stellung des Woinimirs ist aber nichts Genaueres bekannt. ${ }^{26}$ Ebenfalls als bloße Hypothese kann die Identifikation Ingos mit dem im Rhythmus de Pippini regis victoria Avarica auftauchenden Unguimeri betrachtet werden. Unguimeri hatte 796, als das fränkischbayerische Heer sich dem Land der Awaren näherte, dem Awarenkagan und dessen Hauptgemahlin, der Katun ein schreckliches Ende prophezeit. Unguimeri wird oft mit Inguiomer(us), dem Germanenfürsten aus den Annalen des Tacitus (1, 60. 68; 2, 17. 21. 45, 46) in Verbindung gebracht, und deswegen als einer der Gepiden im Awarenreiche angesehen. Walter Pohl meinte, dass Unguimeri ein unter den Awaren lebender Langobarde in der Emigration gewesen sein musste. Andere Forscher setzten Unguimeri wiederum mit dem schon erwähnten Slawen Woinimir gleich, wodurch die Indentifikationsversuche anfingen sich in einem circulus vitiosus zu drehen. ${ }^{27}$

In seiner Ausgabe aus dem Jahre 1979 und in seiner Monografie aus 1995 zieht Herwig Wolfram den Giftzahn der Frage, indem er behauptet, dass Ingo als Herzog sein historisches Dasein nur einem schematischen Übersetzungs-, bzw Interpretationsfehler zu verdanken hat. Das Relativpronomen des Satzanfanges „Quorum unus ... “ wurde nämlich nicht mit den von Arn nach Karantanien und in das untere Pannonien gesandten Glaubensboten, den presbyteri, sondern mit den dort wohnenden Edelsleuten in Verbindung gebracht. ${ }^{28}$ Da die im Dativ stehende Wendung "illis ducibus atque comitibus" dem Satzende näher steht, als der Akkusativ ,presbyteros”, wurde der Subjekt des nächsten Satzes allzu schematisch, sowohl grammatisch, als auch inhaltlich inkorrekt, nicht mit dem direkten Objekt

23 Eisler (Fn 21) S 90.

24 Ingo findet auch als Graf Erwähnung, der sich energisch für die kirchlichen Interessen einsetzte, so zB A Hauck Kirchengeschichte Deutschlands (Berlin-Leipzig, 1954) Bd 2 S 480.

25 M Mitterauer "Slawischer und bayerischer Adel am Ausgang der Karolingerzeit" Carinthia (1960) 150 S 693-726, 695.

26 W Pohl Die Awaren: Ein Steppenvolk in Mitteleuropa 567-822 n. Chr. (München, 1988) S 319-320; Szádeczky-Kardoss (Fn 11) S 286; H Wolfram Die Geburt Mitteleuropas: Geschichte Österreichs vor seiner Entstehung: 378-907 (Wien, 1987) S 258.

27 So zB J Lésny „Unguimer“ in Lexicon Antiquitatum Slavicarum (Wratislawiae, 1977) Bd 6 S 264265; Magnae Moraviae Fontes Historici (Praha-Brno, 1967-1969) Bd 2 S 14; Bd 2 S 305.

28 Wolfram (Fn 8) S 96; Wolfram (Fn 9) S 288. Zu Erzbischof Arn siehe G Demmelbauer Arno, der erste Erzbischof von Salzburg 798-821 (Dissertation, Wien, 1950); M Niederkorn-Bruck \& A Scharer (Hrsg) Erzbischof Arn von Salzburg (Wien-München, 2004). 
des vorherigen Satzes (presbyteros) verbunden. ${ }^{29} \mathrm{Zu}$ Arns Zeiten wurde dieses Gebiet von den duces, dh den Karantnenfürsten und den comites, den Vertretern der fränkisch-bayerischen Herrschaft verwaltet, und die behandelte Stelle betont, dass die von Bischof Virgil auf Karantanien angewandte Missionsmethoden von Arn auf das untere Pannonien übertragen wurden. Wie auch der heilige Rupert nie nach Unterpannonien gelangte, so konnte Virgil auch nicht die Missionstätigkeit auf diesem Gebiet organisieren: diese Behauptungen dienten bloß jenem Zweck, dass die Rechtsansprüche von Salzburg auf Karantanien und das untere Pannonien untermauert werden. Mit dem Satzanfang ,, Simili modo ... ” betont der Verfasser, dass der Leiter des Salzburger Bistums auch in das untere Pannonien Priester entsandte. ${ }^{30}$

Diese Argumentation wird auch durch das Excerptum de Karentanis unterstützt. Das an der Wende des 12 und 13 Jahrhunderts entstandene Excerptum de Karentanis enthält hauptsächlich die Namen der weltlichen und kirchlichen Leitern Karantaniens. Obwohl es alle in der Conversio erwähnten und mit den Karantanen in Verbindung stehenden Namen, außer die der Priester und Diakone, aufzählt, wird ein Herzog, bzw Fürst mit dem Namen Ingo nicht erwähnt. ${ }^{31}$ Diese Liste erstellte der Verfasser aus den Sätzen und Satzteilen der Conversio, aber die Schreibweise der Namen weicht von jener der Conversio ab. Wenn wir uns für die Interpretation ,, ... presbyteros ... Quorum unus ..." entscheiden, müssen wir versuchen, die Existenz eines Priesters namens Ingo zu Arns Zeiten aus den Salzburger Quellen belegen. Es befindet sich in dem Liber confraternitatis, dh dem Verbrüderungsbuch von SanktPeter zu Salzburg ein Eintrag über einen „Ingo presbyter”. ${ }^{32}$ Erwähnenswerts ist zugleich, dass uns außer dem Liber confraternitatis und der Conversio kein Beleg dafür zur Verfügung steht, dass in jener Zeit in Bayern jemand den Namen Ingo getragen hätte. Die besagte Stelle des Salzburger Verbrüderungsbuches erwähnt

29 Die Frage, ob „unus” auf die Priester oder auf die Herzöge und Grafen bezogen werden sollte, warf schon August Jaksch auf. Vgl A Jaksch „Fredegar und die Conversio Carantanorum (Ingo)“ Mitteilungen des Instituts für österreichische Geschichtsforschung (1926) 41 S 44-45.

30 Wolfram (Fn 8) S 97; Wolfram (Fn 9) 228ff. Über Ruperts angebliche Reise nach Pannonien siehe Conversio Bagoariorum et Carantanorum 1; zur Rupertlegende (Gesta sancti Hrodberti confessoris) vgl E Zöllner „Woher stammte der heilige Rupert?“ Mitteilungen des Instituts für österreichische Geschichtsforschung (1949) 57 S 1-22; I Zibermayr „Die Rupertlegende“ Mitteilungen des Instituts für österreichische Geschichtsforschung (1954) 62 S 67-82; H Wolfram „Der heilige Rupert und die antikarolingische Adelsopposition“ Mitteilungen des Instituts für österreichische Geschichtsforschung (1972) 80 S 4-34; H Baltl „Zur Datierungfrage des hl. Rupert“ (1975) 61 Zeitschrift der Savigny-Stiftung für Rechtsgeschichte, Kanonistische Abteilung S 1-16; K Reindel „Die Organisation der Salzburger Kirche im Zeitalter des hl. Rupert“ (1975) 115 Mitteilungen der Gesellschaft für Salzburger Landeskunde S 83-98; J Jahn Ducatus Baiuvariorum: Das bairische Herzogtum der Agilolfinger (Stuttgart, 1991) S 54ff; K Forstner "Quellenkundliche Beobachtungen an den ältesten Salzburger Güterverzeichnissen und an der Vita s. Ruperti“ (1995) 135 Mitteilungen der Gesellschaft für Salzburger Landeskunde S 465-488.

31 Excerptum de Karentanis 2.

32 Liber confraternitatum sancti Petri Salisburgensis vetustior 48. 
Ingo als ersten einer vierzehnköpfigen Gruppe, in der es sich neben jedem Namen im Gegensatz zu den Zeilen im Umfeld - der Zusatz , presbyter ” findet. Ingo musste mit großer Wahrscheinlichkeit der Leiter der Glaubensboten in Karantanien gewesen sein, dessen Wirken in die Zeit zwischen 785 und 799 anzusetzen ist. Wie es im 8 Kapitel Conversio berichtet wird, betraute Arn in 799, ein Jahr nach seiner Erhebung zum Erzbischof einen Chorbischof namens Theoderich mit der Organisation und Leitung der Karantanenmission. Ingo leitete also die Mission in Karantanien beinahe anderthalb Jahrzehnte lang, und die bei der Verrichtung dieser Aufgabe so wichtige prudentia verlieh ihm höchstes Ansehen, wie es aus den zwei, von der Conversio erwähnten Beispielen hervorgeht. Der Lösungsversuch von Herwig Wolfram fand in der Fachliteratur allgemeine Anerkennung, ua pflichtete diesem sowohl Karl Schmid in seiner Studie über das Verbrüderungsbuch, als auch Fritz Lošek in seiner Conversioausgabe bei. ${ }^{33}$

4. In der Erzählung der Conversio sollte Ingo ein Blatt, bzw Pergamen ohne Schrift (carta sine litteris) geschickt haben, das für seinen Boten genügend Legitimation gab, vom Volke Gehorsam zu verlangen. Die carta als Urkunde von größter Beweiskraft wurde zwar von den Germanen in ihr Rechtssystem aus dem römischen Recht übernommen, erreichte jedoch, weil ihnen einerseits das Latein der Urkunden, andererseits die Schreib- und Lesekunst nur in geringem Maße zugänglich war, bei ihnen kein allzu hohes Ansehen, dh mit dem Rezeptionsakt nahm zugleich der Abweisungsprozess seinen Anfang. ${ }^{34}$ Im Zusammenhang mit dieser Tatsache ist es festzulegen, dass im Falle der von Ingo gesandten carta das Fehlen der Schrift auch deswegen nicht als überraschender Moment überbewertet werden dürfte, weil die neu bekehrten Slawen und Awaren des Lesens unkundig waren, wie es auch aus dem Protokoll des Conventus episcoporum ad ripas Danubii aus dem Jahre 796 hervorgeht. ${ }^{35}$

Im fränkischen, bayerischen, alemannischen und burgundischen Rechtswesen wurde eine ziemlich seltsame Art des urkundlichen Beweisverfahrens ausgearbeitet. Die Urkunde, oder genauer gesagt das leere Pergament, das eben durch die Beschriftung zur Urkunde werden sollte, entwickelte sich zu einem bei Liegenschaftsübertragungen gebräuchlichen Symbol. Beim Rechtsakt wurde es auf die Erde gelegt, und der Aussteller der Urkunde hob es während der entsprechenden Willensäußerung von der Erde auf, und überreichte es dem Schreiber. Anlässlich

33 Wolfram (Fn 8) S 98f; K Schmid „Das Zeugnis der Verbrüderungsbücher zur Slawenmission“ in Th Piffl-Perčević \& A Stirnemann (Hrsg) Der heilige Method, Salzburg und die Slawenmission (Innsbruck-Wien, 1987) S 185-205, 188; Lošek (Fn 8) S 112.

34 Vgl M Kos „Carta sine litteris“ (1954) 62 Mitteilungen des Instituts für österreichische Geschichtsforschung S 97-100, 98.

35 F Zagiba „Die Missionierung der Slaven aus,Welschland“ (Patriarchat Aquileia) im 8. und 9. Jahrhundert" in M Hellmann, R Olesch, B Stasiewski \& F Zagiba (Hrsg) Cyrillo-Methodiana. Zur Frühgeschichte des Christentums bei den Slaven 863-1963 (Graz, 1964) S 273-311, 280. 
der Forderung, dass das Pergament mit der Erde in Berührung kommen sollte, ist als Parallele aus dem römischen Recht die Eigenheit der mancipatio und der legis actio sacramento in rem herauszuheben, dass die Parteien mit Hilfe eines Stabes den Gegenstand des Rechtsaktes, bzw des Rechtsstreites haben berühren müssen. Die Berührung kann nicht ausschließlich zur genaueren Bestimmung gedient haben, denn dazu hätte eine eindeutige Hindeutung gereicht, der Berührungsakt erschuf in den archaischen, mit dem Sakralen noch engere Verbindungen hegenden Rechtsordnungen eine stärkere Bindekraft und die Möglichkeit der Willensübertragung. ${ }^{36}$ Das Blatt konnte manchmal auch schon im Voraus, gleichsam formelhaft ausgefüllt werden, aber die Siegel der Zeugen, die Unterschrift des Schreibers und die Datierung wurden erst suo tempore zum Pergament gebracht - solange galt die Urkunde de iure als ungeschrieben, da sie den notwendigen Gültigkeitskriterien nicht entsprach. Die Symbolhaftigkeit der carta wurde in manchen Fällen auch dadurch verstärkt, dass neben das Pergament auch der Tintenfass und die Feder auf die Erde gelegt wurde, und der Aussteller diese Gegenstände zusammen mit dem Pergament von der Erde aufheben, und dem Schreiber übergeben musste. ${ }^{37}$ Herwig Wolfram stellt die Frage, ob ein leeres Pergament, wie auch in der Geschichte des Ingo, ausreichende Beweiskraft besaß, und es noch nicht irgendwelche Zeichen (signa) und Siegel erforderlich waren. Das Missachten des herzoglichen Siegels wurde bekanntlich sowohl vom alemannischen, als auch vom bayerischen Recht sanktioniert. ${ }^{38}$

Der Gegenstand, das Symbol, das unausgefüllte, dh noch nicht zur carta gewordene Pergament hatte in manchen Fällen mehr Wert, als die schon ausgestellte, gültige Urkunde, die sowieso nur wenige zu lesen vermochten. Die Erzählung der Conversio macht eben die Tatsache deutlich, dass die bloße Versendung des Pergaments, das entsprechend ausgestellt erst später zur rechtmäßigen Urkunde hätte werden können, ausreichte, die gewünschte Wirkung beim Volke zu erzielen. Diesen Verhältnissen entsprach der Brauch vollkommen, dass der Eigentumsübertragung nicht die ausgefüllte carta, sondern die, mit der Willenserklärung gleichzeitig verlaufende Zeremonie, bei der das Pergament von der Erde aufgehoben und dem Schreiber überreicht wurde, ihre Gültigkeit verlieh. In der des Schreibens und des Lesens unkundigen Umgebung diente das Pergament, das das Material für die Urkunde lieferte als ausreichendes Beweismittel. Genauso ,sprach” der Siegel selbst für diejenigen, die dessen Schriftzeichen nicht lesen konnten, eine wohl verständliche Sprache. In der Formulierung von Milko Kos bedeutete die carta sine litteris nichts anderes: ,Ingo sandte mich, gehorche meinem Befehl!’. Als Analogie erwähnte er

36 Vgl A Hägerström Der römische Obligationsbegriff im Lichte der allgemeinen römischen Rechtsanschauung (Uppsala, 1927) Bd 1 S 33-41; T Nótári „Comments on the origin of the legis actio sacramento in rem“ (2006) 47 Acta Juridica Hungarica S 133-155.

37 O Redlich „Privaturkunden des Mittelalters“ in W Erben, L Schmitz-Kallenberg \& O Redlich Urkundenlehre (München, 1911) Bd 3 S 47; E Goldmann „Cartam levare“(1914) 35 Mitteilungen des Instituts für österreichische Geschichtsforschung S 1-59; Kos (Fn 34) S 99.

38 Wolfram (Fn 8) S 199; Leges Alamannorum 22, 2; Lex Baiuvariorum 2, 13. 
das Siegel von Otto von Trixen aus dem 12. Jahrhundert, auf dem folgender Satz zu lesen war: „Otto de Trussen me misit”. ${ }^{39}$

Aufgrund all dessen ist es mehr oder weniger irrelevant, ob wir das Versenden des Pergaments durch Ingo als einen historischen Fakt, oder als eine Parabel, die sein Ansehen zu demonstrieren hatte, bewerten, denn irgendwie musste diese Erzählung über die carta sine litteris den Verhältnissen des 12 Jahrhunderts entsprechen, ansonsten wäre es für den Adressaten und den anderen Lesern der Conversio unverständlich geblieben. Es ist eben den besagten Schwierigkeiten bei der Interpretation zuzuschreiben, dass das Moment der carta sine litteris, zu dessen Verständnis das Vertrags- und Urkundensystem des frühmittelalterlichen germanischen Rechtswesens von Nöten ist, für die Autoren späterer Zeiten keinen lehrreichen und für eine Erzählung geeigneten Stoff bot. So fand die carta sine litteris weder Eingang in den Liber certarum historiarum des Johanns von Viktring, noch in die De Europa des Enea Silvio Piccolomini - im Gegensatz zur Geschichte über Ingos Gastmahl.

5. Die Erzählung über Ingos Gastmahl in der Conversio Bagoariorum et Carantanorum ist ihrer Literaturgattung nach als christliche Parabel einzuordnen, in ihrer Struktur folgt sie auch den biblischen Vorbildern: in ihr sollte den schon Bekehrten der Erfolg der Mission in Karantanien geschildert werden. Der Gastgeber erweist den Christen die Ehre, selbst wenn sie Knechte sein sollten, ihnen das Mahl in goldenen Gefäßen aufzutischen, während dessen müssten die Ungläubigen, und sollten sie selbst die mächtigsten Herren der Welt sein, vor den Türen aus schmutzigen Tonschüsseln wie die Hunde speisen. Das Fazit der Geschichte steht im deutlichen Einklang mit der Erzählung über das Königsmahl aus dem Matthäusevangelium (22, 1-14), bei dem diejenigen, die nicht im Festgewand erscheinen, in die Dunkelheit hinausgestoßen werden, wo ihnen nichts anderes übrig bleibt, als zu weinen, zu jammern und die Zähne zu knirschen. Das Christentum macht selbst die Knechte zu Adeligen, während die ungläubigen Herren ihr Leben in Schmutz und in Knechtschaft der Sünde zubringen müssen. Für die Parabel des ingonischen Gastmahles lassen sich auch aus der Literatur ihrer Zeit Parallele finden, so zB wird uns eine ähnliche Geschichte über den im Hofe des Skatopluk in Moravien lebenden Fürsten Bořivoj überliefert. ${ }^{40}$ Aufmerksamkeit verdient auch eine Textstelle bei Fredegar, auf die schon Arnold Jaksch als mögliches Vorbild der Erzählung in der Conversio hingewiesen hat. ${ }^{41}$

Sowohl in der Erzählung über Ingos Gastmahl in der Conversio, als auch in der Geschichte bei Fredegar werden die noch ungetauften Heiden als Hunde bezeichnet,

39 Kos (Fn 34) S 99f; Redlich (Fn 37) Bd 3 S 108.

40 Legenda Christiani: vita et passio sancti Wenceslai et sanctae Ludmillae avae eius 1-3. Zu der Verbindung mit der Conversio siehe J Pekař Die Wenzels- und Ludmilla-Legenden und die Echtheit Christians (Prag, 1906) S 88ff.

41 Jaksch (Fn 29) S 154. 
bzw beschimpft. In Fredegars Chronik $(4,68)$ sagt der sich wegen der Niedermetzelung der fränkischen Gesandten beklagende Königsbote Sicharius dem Fürsten Samo, dass er es für unmöglich hielte, dass die Diener Gottes mit den Hunden zusammen speisten. Darauf entgegnet Samo, dass, wenn sie schon als Hunde beschimpft worden wären, ihnen das Recht zustehen würde ihren Feinden Bisse zuzufügen. An diesem Punkt vollzieht sich jedoch ein Aspektwandel: Während der Verfasser der Conversio, der die fredegarischen Werke kennt und gebraucht, den Ingo die ungetauften Herren vor die Tür setzen lässt, wird bei Fredegar der Sicharius, ein Christ und Gesandter des Frankenkönigs vom Heiden Samo zur Tür hinausgeworfen:

Eo anno Sclavi coinomento Winidi in regno Samone neguciantes Francorum cum plure multetudine interfecissent et rebus expoliassint, haec fuit inicium scandali inter Dagobertum et Samonem regem Sclavinorum. Dirigensque Dagobertus Sycharium legatarium ad Samonem, paetens, ut neguciantes, quos sui interfecerant aut res inlecete usorpaverant, cum iusticia faceret emendare. Samo nolens Sicharium vedere, nec ad se eum venire permitteret, Sicharius vestem indutus ad instar Sclavinorum, cum suis ad conspectum pervenit Samonem [...] Sicharius dicens: 'Non est possebelem, ut christiani et Dei servi cum canebus amicicias conlocare possint. 'Samo a contrario dixit: 'Si vos estis Dei servi, et nos Dei canes, dum vos adsiduae contra ipsum agetis, nos permissum accepimus vos morsebus lacerare.' Aegectus est Sicharius de conspectum Samonis. ${ }^{42}$

Die Vorbildfunktion der Fredegar-Stelle wird auch dadurch untermauert, dass das hier von uns behandelte 7 Kapitel der Conversio nicht der einzige gemeinsame Punkt zwischen beiden Werken ist. In dem sich mit Samo befassenden Teil des 4 Kapitels der Conversio wird reichlich aus Fredegars Chronik geschöpft. Aus den angeführten Stellen wird eindeutig ersichtlich, dass Fredegars Erzählung die Ereignisse aus einem vollkommen anderen Blickwinkel schildert, als die Conversio. Laut Fredegar sollte der Franke Samo in 623/24 als Waffenhändler und vielleicht als Beauftragter des Frankenkönigs Dagobert I (623-639) unter die Slawen gegangen sein, um sie bei ihren Selbstständigkeitsbestrebungen zu unterstützen. Die Krise des durch die Besetzungsniederlage bei Byzanz im Jahre 626 geschwächten ersten Awarenkaganats ermöglichte Samo sich zum Fürsten eines von ihm gegründeten mitteleuropäischen Staates erheben zu lassen. Diesen Staat wollte König Dagobert I vernichten, seine Versuche blieben jedoch erfolglos, und somit löste sich der besagte Staat erst mit dem Tode des Samo in 658 auf. Laut der Conversio sollte Samo ein dux gentis karantanischer Herkunft gewesen sein, und das Heer des Dagoberts I hätte die Rebellion der Slawen erfolgreich niedergeschlagen. Um seine eigene Version schreiben zu können musste aber der Verfasser der Conversio nicht Fredegars Chronik, sondern die auf Fredegar zurückgehende Gesta Dagoberti I. regis Francorum als Vorlage gebrauchen. So wurde der Fürst des Slawenstaates Samo 
selber zum Slawen, und die von den Franken und Langobarden angegriffene Staat mit Karantanien indentifiziert. ${ }^{43}$

Ohne bei dieser literarischen Vorlage der Conversio (und zugleich bestem morphologischen Paradebeispiel des Lateins der Merowingerzeit) aus dem 7 Jahrhundert länger verweilen zu müssen, lässt es sich feststellen, dass die Erzählung über Ingos Gastmahl als Teil des durch die Slawen- und Awarenmission notwendig gewordenen Missionskatechismus der Karolingerzeit entstand, ${ }^{44}$ Diese Parabel wirkte über Jahrhunderte hindurch fort, und wurde selbst im 14 Jahrhundert von Johann von Viktring und im 15 Jahrhundert von Enea Silvio Piccolomini als ein charakteristisches Beispiel des Kulturgutes des ausgehenden 8 Jahrhunderts geschätzt.

6. Als ursprüngliche Quelle der Erzählung über Ingos Gastmahl im 65 Kapitel des Werkes De Europa von Enea Silvio Piccolomini kann der 7 Kapitel der in 870 entstandenen Conversio Bagoariorum et Carantanorum gelten. Obwohl Enea Silvio die Conversio gekannt und gelesen haben muß, ist die Geschichte von Ingo, wo er von dem Weißbuch der Salzburger Kirche als Quelle Gebrauch macht. Festgestellt werden konnte auch, dass in der Conversio Ingo nicht als Herzog oder Graf auftritt, und dank der interpretatio grammatica und der Parallelstelle des Salzburger Verbrüderungsbuches war auch zu ermittelt, dass er in der Zeit zwischen 785 und 799 der von Arn beauftragte Leiter der Karantanenmission tätig war. Sein Dasein als Herzog verdankt Ingo einer Fehlinterpretation, die zum ersten Mal am Anfang des 14 Jahrhunderts in dem Liber certarum historiarum des Abtes Johann von Viktring anzutreffen ist. Diese missglückte Deutung übernahm die Literatur von mehreren Jahrhunderten, und so versuchten manche Forscher den angeblichen Herzog Ingo mit einer tatsächlich belegbaren historischen Gestalt zu identifizieren zu.

Die Geschichte des ingonischen Gastmahles weist neben den biblischen Parallelen einige Verbindungen mit der Chronik des Fredegars auf. Der Verfasser der Conversio verwendete das bei Fredegar gefundene Motiv zu seinen eigenen Zwecken, und fügte es harmonisch in seine Erzählung hinein - eine Erzählung, die sich hervorragend als Parabole der Slawen- und Awarenmission eignete. Die Tatsache, dass Enea Silvio, ein Kenner des Werkes des Johanns von Viktring über

43 Conversio Bagoariorum et Carntanorum 4. Temporibus gloriosi regis Francorum Dagoberti Samo nomine quidam Sclavus manens in Quarantanis fuit dux gentis illius. Qui venientes negotiatores Dagoberti regis interficere iussit et regia expoliavit pecunia. Quod cum comperit Dagobertus rex, misit exercitum suum et damnum, quos ei idem Samo fecit, vindicare iussit. Hierzu vgl die Stelle der Fredegar Chronik des Fredegar $(4,48)$ und jene der Gesta Dagoberti I. regis Francorum (27), zu ihrer Analyse siehe Szádeczky-Kardoss (Fn 11) S 171; Pohl (Fn 26) S 256; Lošek (Fn 8) S 31. Zu den anderen Aspekten siehe J Goll „Samo und die karantanischen Slaven“ (1890) 11 Mitteilungen des Instituts für österreichische Geschichtsforschung S 441-446.

44 H Löwe Die karolingische Reichsgründung und der Südosten: Studien zum Werden des Deutschtums und seiner Auseinandersetzung mit Rom (Stuttgart, 1937) S 119, 170. 
Ingo den Karantanenherzog schrieb, lässt uns annehmen, dass er die viktringische Interpretation der von ihm selber auch gelesenen Stell der Conversio akzeptiert hatte. Es ist allzu unwahrscheinlich, dass Enea Silvio von Johann von Viktring unabhängig zur selben Fehldeutung des Textes gelangt wäre. Der Wertunterschied zwischen Christen und Ungläubigen zeigt sich in allen von uns hier untersuchten Erzählungen in der Relation des Drinnen und Draußen, in der Dualität des Einlasses und des Aussperrens. Der Vergleich der Heiden mit den Hunden ist jedoch bei Enea Silvio und Iohannes Victoriensis nicht mehr zu finden. Bei Fredegar lässt sich das Hundemotiv zwar antreffen, wird aber mit einer Parabel über ein Gastmahl in keinerlei Verbindung gebracht, da es ja in der Fredegarstelle nicht um ein Gastmahl, sondern um das Verantworten der Niedermetzelung fränkischer Gesandten und Händlern geht. Die besagten Motive werden nur in der Conversio und im Liber certarum historiarum miteinander kombiniert. Jener Satz der Conversio, der besagt, das Volk hätte Ingo selbst dann gehorcht, wenn er ihnen ein leeres Pergament, eine carta sine litteris zugesandt hätte, stieß bei Enea Silvio und Johann von Viktring wahrscheinlich auf Interpretationsschwierigkeiten wegen der allzu organischen Verbindung des Bildes mit dem frühmittelalterlichen Urkundenwesen, und fand weder in die De Europa, noch in den Liber certarum historiarum Eingang.

\section{ABSTRACT}

Enea Silvio Piccolomini, in his work entitled De Europa written in 1458, tells an interesting story defined as a legend in terms of genre about a duke called Ingo, who lived during the reign of Charlemagne. This narrative claims that in $790 \mathrm{dux}$ gentis Ingo held a feast for the inhabitants of his province where food was served in golden and silver bowls to the peasants allowed to appear before him, while to the dignitaries standing further away from him received their food in bowls made of clay. The researchers' attention is deservedly raised by the question why this parabolic story with biblical tone was included in Enea Silvio's work; and if it had been borrowed, from whom? The answer seems to be very simple: it derives from the Conversio Bagoariorum et Carantanorum drafted regarding the lawsuit instituted Methodius. In the case narrated in the Conversio Ingo sent a charter, or rather a parchment without any writing or letters on it (carta sine litteris), which provided his legate with sufficient authenticity to demand obedience from the people.

In this study - after having compared the two narratives and outlined the place of De Europa in Enea Silvio Piccolomini's oeuvre as well as the circumstances of the drafting and tendencies of the Conversio Bagoariorum et Carantanorum, the author attempts to answer the following questions: To what extent can duke Ingo, mentioned by Enea Silvio and not questioned in the literature for long centuries, be considered a real historical person? Does the Conversio refer to Ingo as a duke, and if it does, what is his existence as a duke and introduction in the literature as a duke 
owing to? What could the meaning of carta sine litteris referred to in Conversio have been, and why did Enea Silvio not take this item over although he could have put it forward as a further proof of Ingo's dignity? To what literary prefigurations can the description of the feast held by Ingo be traced back to, and what role did it play in the Conversio? And, regarding the borrowing of the Ingo story by Enea Silvio, what possible intermediary writing and author can be reckoned with? 